\title{
NBS
}

Eechnical Mote

\section{AN EVALUATION OF KACSER'S SECOND ORDER BORN APPROXIMATION TO THE} BREMSSTRAHLUNG DIFFERENTIAL CROSS SECTION 


\section{THE NATIONAL, BUREAU OF STANDARDS}

\section{Functions and Activities}

The funetions of the National Bureau of Standards are set forth in the Act of Congress, March 3, 1901, as amended by Congress in Public Law 619, 1950. These inelude the development and maintenance of the national standards of measurement and the provision of means and methods for making measurements consistent with these standards; the deterinination of physical constants and properties of materials; the development of methods and instruments for testing materials, devices, and structures; advisory services to government agcncies on scientific and technical problcins; invention and devclopment of devices to serve special needs of the Government; and the development of standard practices, codcs, and specifications. The work includes basic and applied research, development, engineering, instrumentation, testing, evaluation, calibration services, and various consultation and information services. Researeh projects are also performed for other government ageneies when the work relates to and supplements the basic program of the Bureau or when the Bureau's unique competence is required. The scope of activities is suggested by the listing of divisions and sections on the inside of the back cover.

\section{Publications}

The results of the Bureau's work take the form of either actual cquipment and devices or published papers. These papers appear either in the Burean's own series of publications or in the journals of professional and scientific societies. The Bureau itsclf publishes three periodicals available from the Government Printing Office: The Journal of Research, published in four separate sections, presents complete scientific and technical papers; the Technical News Bulletin presents sumunary and preliminary reports on work in progress; and Basir Radio Propagation Predictions provides data for determining the best frequencies to use for radio communications throughout the world. There are also five series of nonperiodical publications: Monographs, Applied Mathematics Series, Handbooks, Miscellaneous Publications, and Technical Notes.

Information on the Bureau's publieations can be found in NBS Circular 460, Publications of the National Bureau of Standards (\$1.25) and its Supplement $(\$ 1.50)$, available from the Superintendent of Documents, Government Printing Office, Washington 25, D.C. 


\title{
NATIONAL BUREAU OF STANDARDS Eechnical Note
}

81

JUNE 1961

\section{AN EVALUATION OF KACSER'S SECOND ORDER BORN APPROXIMATION TO THE BREMSSTRAHLUNG DIFFERENTIAL CROSS SECTION}

\author{
George S. Ofelt
}

NBS Technical Notes are designed to supplement the Bureau's regular publications program. They provide a means for making available scientific data that are of transient or limited interest. Technical Notes may be listed or referred to in the open literature. They are for sale by the Office of Technical Services, U. S. Department of Commerce, Washington 25, D. C.

\section{DISTRIBUTED BY \\ UNITED STATES DEPARTMENT OF COMMERCE OFFICE OF TECHNICAL SERVICES \\ WASHINGTON 25, D. C.}

Price $\$ 0.75$ 

An Evaluation of Kacser's Second Order Born Approximation to the Bremsstrahlung Differential Cross Section

\section{George S. Ofelt}

The second order term, as derived by C. Kacser [Proc. Roy. Soc. A253 (1959)], of the Born approximation series for the bremsstrahlung cross section differential with respect to photon energy, photon direction, and final electron direction averaged over initial and summed over final polarization states has been put in a form that admits to numerical evaluation for the coplanar case. The results are valid for relativistic as well as non-relativistic incident electrons.

Completely general (non-coplanar) expressions are included for the first order (Bethe-Heitler) and second order terms. However, the latter has not been given in a form admissible to numerical evaluation.

Tabulated values are given for the case of the incident electron kinetic energy $=500 \mathrm{kev}$, photon energy $=450 \mathrm{kev}$, and photon direction $20^{\circ}$ from the incident electron direction. For this case the second order term increases the differential cross section by about 50 percent for $\mathrm{Al}$ and about 200 percent for $\mathrm{Au}$ for the various angles of electron emission. A rough approximation to the integrated (over final electron direction) cross section is included and a comparison is made with experimental values. The inclusion of the second order term brings the theoretical cross section closer to the experimental values.

\section{Introduction}

Measurements of the bremsstrahlung cross section, differential with respect to photon energy and direction in the region where the incident electron's kinetic energy is of the order of the electron rest energy, show the predictions of the first order Born approximation theory to be too low by a considerable factor $[1,2]$. This discrepancy increases with increasing atomic number of the scattering material and also with increase of photon energy for a given incident electron energy. The inclusion of screening effects does not remove the discrepancy. At extreme relativistic incident electron energies the effect is reversed--Born approximation theory overestimates the experimental 
values. Therefore, the second order term of the Born approximation series should be evaluated.

The Born approximation is assumed to be valid for the following simultaneous conditions on the initial, $\beta_{0}$, and final, $\beta$, electron velocity ratio to the velocity of light:

$$
\frac{2 \pi \alpha Z}{\beta_{0}}<1 \text { and } \frac{2 \pi \alpha Z}{\beta}<1
$$

which are not satisfied for high values of $Z$. However, the first order Born theory gives at least order of magnitude estimates of the cross section for high values of $\mathrm{Z}$. Therefore a second order calculation might be expected to give reasonable results.

Kacser [3] has calculated the second order term of the Born approximation series for the differential cross section with no screening corrections which is averaged over initial and summed over final polarization states.

The purpose of this paper is to give the second order term, as derived by Kacser, in a form that is readily calculable. In particular only the coplanar case--final electron momentum vector lying in the plane defined by the initial electron momentum and the photon momentum vectors-is considered.

The cross section obtained is differential with respect to photon energy, photon direction, and final electron direction. Therefore an integration over the final electron direction's solid angle would have to be performed in order to compare the contribution of this second order term with existing experimental results. However, this cannot be done easily since only the coplanar case is reduced to a reasonable form. A rough indication of the magnitude of this is given in section 8 (for the tabulated case of section 7).

2. Symbols, Constants, and Energy-Momentum Relations The system of units and symbols used follow those of Koch and Motz [2] with new symbols being introduced as needed. $\mathrm{T}_{\mathrm{o}}=$ incident electron kinetic energy in $\mathrm{mc}^{2}$ units. $\mathrm{E}_{\mathrm{O}}=$ incident electron total energy in $\mathrm{mc}^{2}$ units. $\mathrm{E}=$ final electron total energy in $\mathrm{mc}^{2}$ units. 
$\mathrm{k}=$ photon energy in $\mathrm{mc}^{2}$ units.

$\vec{P}_{0}=$ incident electron momentum in mc units.

$\overrightarrow{\mathrm{P}}$ = final electron momentum in mc units.

$\overrightarrow{\mathrm{k}}$ = photon momentum in mc units.

$\vec{\tau} \equiv \overrightarrow{\mathrm{P}}_{0}-\overrightarrow{\mathrm{k}}$

$\overrightarrow{\mathrm{q}} \equiv \vec{\tau}-\overrightarrow{\mathrm{p}}$ momentum transfer to nucleus.

$\theta_{0}=$ angle between $\vec{P}_{0}$ and $\vec{k}$ in general case.

$\varphi_{0}=$ angle between " and "in coplanar case.

$\theta=$ angle between $\vec{P}$ and "in general case.

$\varphi$ = angle between " and "in coplanar case.

$\phi=$ angle between the planes $\left(\vec{P}_{0}, \vec{k}\right)$ and $(\vec{P}, \vec{k})$.

$\chi \equiv \varphi_{0}-\varphi$.

$\mathrm{d} \Omega_{\mathrm{k}}=$ element of solid angle in direction of $\vec{k}$.

$\mathrm{d} \Omega_{\mathrm{p}}=$ element of solid angle in direction of $\overrightarrow{\mathrm{P}}$.

$r_{0}=2.82 \times 10^{-13} \mathrm{~cm}$ classical electron radius.

$\alpha=\frac{1}{137}$ fine structure constant.

$\mathrm{Z}$ = atomic number of target material.

$\mathrm{m}_{\mathrm{o}} \mathrm{c}^{2}=.511 \mathrm{Mev}$ electron rest energy.

$E_{0}=T_{0}+1$.

$E=E_{0}-k_{0}$

$P_{0}=\left(E_{0}^{2}-1\right)^{1 / 2}$.

$P=\left(E^{2}-1\right)^{1 / 2}$.

$\beta_{0}=\frac{P_{0}}{E_{0}}$ initial electron velocity's ratio to velocity of light.

$\beta=\frac{P}{E}$ final electron velocity's ratio to velocity of light.

Symbols Used Only in Section 5-A

$\mathrm{n}^{\mu}=$ unit time like 4 vector $(0,0,0,1)$.

$g^{\mu \nu}= \begin{cases}1 & \mu=v=1,2,3 . \\ 0 & \mu \neq v \\ -1 & \mu=v=4 .\end{cases}$

$P_{0}=\left(\vec{P}_{0}, E_{0}\right)$

$\mathrm{P}_{\mathrm{O}}^{\mathrm{i}}=$ ith component of $\overrightarrow{\mathrm{P}}_{\mathrm{O}}$.

$\left(P_{0} P\right)=\left(\vec{P}_{0} \cdot \vec{P}-E_{0} E\right)$ s calar product.

$\left(\mathrm{P}_{\mathrm{O}} \mathrm{P}\right)=\left(\overrightarrow{\mathrm{P}}_{0}^{\circ} \cdot \overrightarrow{\mathrm{P}}_{\mathrm{O}} \mathrm{E}_{0} \mathrm{E}\right)$.

$\left(P_{0} n\right)=-E_{0}$. 
Symbols Defined in Text

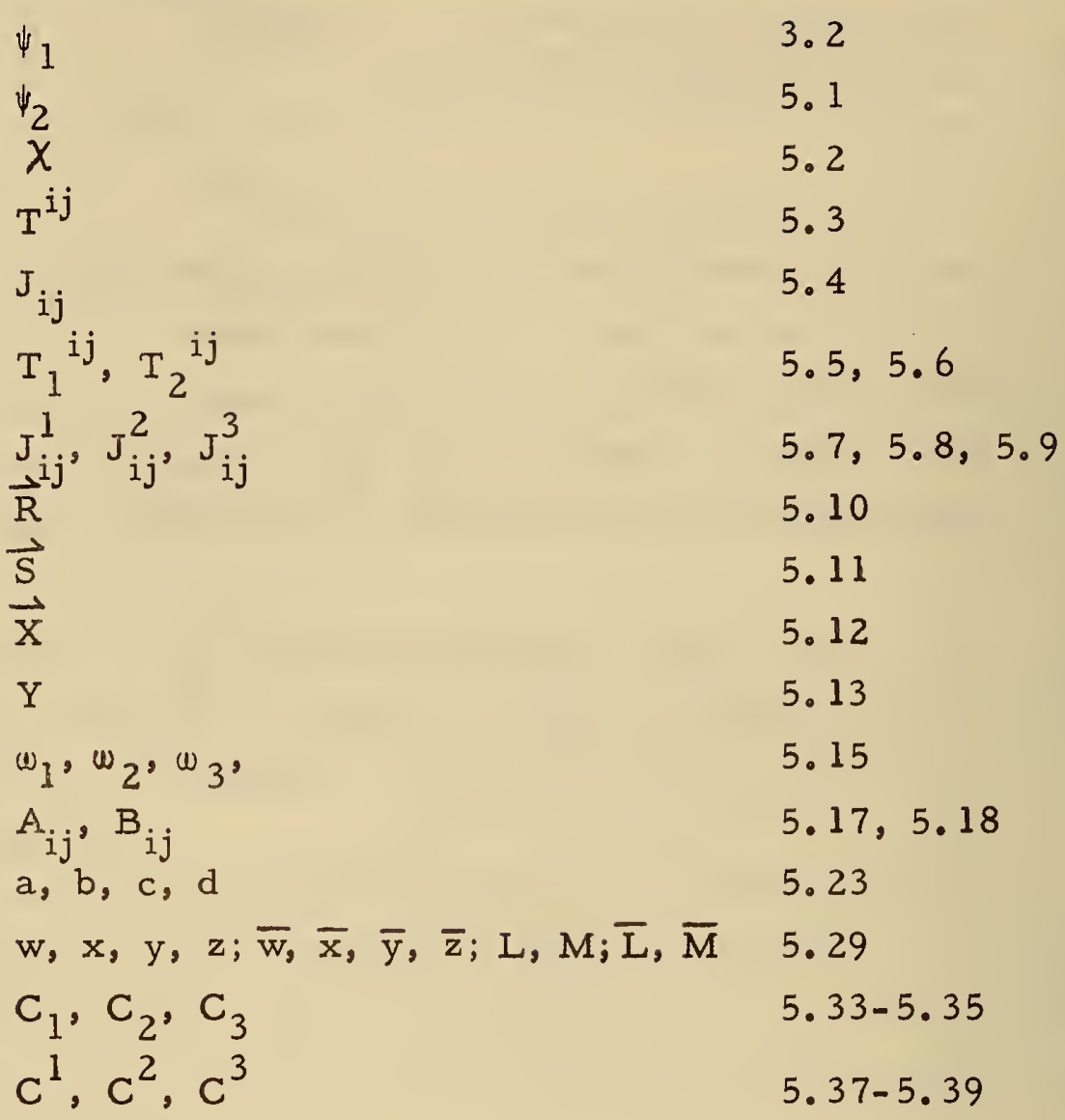

3. General Eormulation for the Differential Cross Section Formula The Born approximation development of the differential cross section in a power series of $\alpha Z$ can be expressed by the following:

$$
\mathrm{d} \sigma=\left[\psi_{1}+\mathrm{Z} \psi_{2}+\cdots \text { H. T. }\right] \mathrm{z}^{2} \frac{\mathrm{dk}}{\mathrm{k}} \mathrm{d} \Omega_{\mathrm{k}} \mathrm{d} \Omega_{P}
$$

where the $\psi^{\prime}$ 's are defined to be independent of $Z$. Therefore the $\psi^{\prime}$ 's are functions of $\mathrm{E}_{\mathrm{o}}, \mathrm{k}, \theta_{\mathrm{O}}, \theta$, and $\phi$

$$
\psi_{1}=\frac{\mathrm{d} \sigma_{1}}{\mathrm{z}^{2} \frac{\mathrm{dk}}{\mathrm{k}} \mathrm{d} \Omega_{\mathrm{k}} \mathrm{d} \Omega_{\mathrm{p}}}
$$

where $d \sigma_{1}$ is the Bethe-Heitler cross section (first order) term, then $\psi_{2}$ is second order in relation to $\psi_{1}$ and is the quantity to be calculated in this paper. 


\section{The First Order Term (Bethe-Heitler)}

A. General Case

This term is given in the same form as in reference (2)。

$$
\begin{aligned}
\psi_{1} & =\frac{\alpha r_{0}^{2}}{(2 \pi)^{2}} \frac{P}{P_{0} q^{4}}\left\{\frac{P^{2} \sin ^{2} \theta}{(E-P \cos \theta)^{2}}\left(4 E_{0}^{2}-q^{2}\right)\right. \\
& +\frac{P_{0}^{2} \sin ^{2} \theta_{0}}{\left(E_{0}-P_{0} \cos \theta_{0}\right)^{2}}\left(4 E^{2}-q^{2}\right) \\
& +\frac{2}{(E-P \cos \theta)\left(E_{0}-P_{0} \cos \theta_{0}\right)}\left[k^{2}\left(P^{2} \sin ^{2} \theta+P_{0}^{2} \sin ^{2} \theta_{0}\right)\right. \\
& \left.\left.-P P_{0} \sin \theta_{\sin \theta_{0}}\left(\left(4 E E_{0}-q^{2}\right)+2 k^{2}\right) \cos \phi\right]\right\}
\end{aligned}
$$

where

$$
\begin{aligned}
q^{2} & =\left\{P_{0}^{2}+P^{2}+k^{2}-2 P_{0} k \cos \theta_{0}+2 P k \cos \theta\right. \\
& \left.-2 P_{0} P\left(\cos \theta_{0} \cos \theta+\sin \theta_{0} \sin \theta \cos \phi\right)\right\}
\end{aligned}
$$

$\vec{k}$ is taken as the polar axis and the angle $\phi$ is determined by which side of $\vec{k}$ the projection of $\vec{P}$ on the $\vec{P}_{0}, \vec{k}$ plane lies. $\frac{\pi}{2}>\phi \geqslant 0$ if the projection of $\overrightarrow{\mathrm{P}}$ lies on the same side of $\overrightarrow{\mathrm{k}}$ as $\overrightarrow{\mathrm{P}}_{\mathrm{O}}$ and $\pi \geq \phi>\frac{\pi}{2}$ if on the opposite side; $\left(\pi \geq \theta_{0} \geq 0, \pi \geq \theta \geq 0\right)$.

B. The Coplanar Case, $\overrightarrow{\mathrm{P}}_{\mathrm{o}} \cdot(\overrightarrow{\mathrm{P}} \times \overrightarrow{\mathrm{k}})=0$

In this case $\phi$ is either zero or $\pi$. We define the angles $\varphi_{0}$ and $\varphi$ in the photon emission plane as the angles between $\vec{P}_{0}$ and $\vec{k}$, and $\vec{P}$ and $\vec{k}$ respectively $\left(2 \pi \geqslant \varphi_{0} \geqslant 0,2 \pi \geqslant \varphi \geqslant 0\right)$. Therefore equation 4.1 becomes:

$$
\begin{aligned}
\psi_{1} & =\frac{\alpha}{(2 \pi)^{2}} r_{0}^{2} \frac{P}{P_{0} q^{4}}\left\{\frac{P^{2} \sin ^{2} \varphi}{(E-P \cos \varphi)^{2}}\left(4 E_{0}^{2}-q^{2}\right)\right. \\
& +\frac{P_{0}^{2} \sin ^{2} \varphi_{0}}{\left(E_{0}-P_{0} \cos \varphi_{0}\right)^{2}}\left(4 E^{2}-q^{2}\right) \\
& +\frac{2}{(E-P \cos \varphi)\left(E_{0}-P_{0} \cos \varphi_{0}\right)}\left[k^{2}\left(P^{2} \sin ^{2} \varphi+P_{0}^{2} \sin ^{2} \varphi_{0}\right)\right. \\
& \left.\left.-P_{0} P \sin \varphi_{0} \sin \varphi\left(\left(4 E_{0} E-q^{2}\right)+2 k^{2}\right)\right]\right\}
\end{aligned}
$$


and 4.2 becomes:

$$
q^{2}=\left\{P_{0}^{2}+P^{2}+k^{2}-2 P_{0} k \cos \varphi_{0}+2 P k \cos \varphi-2 P_{0} P \cos X\right\}
$$

5. The Second Order Term

A. General Case

In the units used here Kacser's second order term* is given by $\psi_{2}=\pi \alpha\left(\frac{E}{P}-\frac{E_{0}}{P_{0}}\right) \psi_{1}+\frac{\alpha^{2}}{2 \pi} r_{0}^{2} \frac{P_{0}^{2}}{P_{0} q^{2}} \chi$

where $\psi_{1}$ is the first order quantity defined in section 4 and $\chi_{\text {is defined }}$

$$
X=\left[\frac{2}{\pi^{3}} \mathrm{~T}^{\mathrm{ij}} \mathrm{J}_{\mathrm{ij}}+\frac{1}{(\mathrm{Pk})} \overrightarrow{\mathrm{R}} \cdot \overrightarrow{\mathrm{X}}-\frac{1}{\left(\mathrm{P}_{\mathrm{O}} \mathrm{k}\right)} \overrightarrow{\mathrm{S}} \cdot \overrightarrow{\mathrm{Y}}\right]
$$

In order to facilitate calculations in the coplanar case $T^{i j}$ and $J_{i j}$ are defined in several parts

$$
\begin{aligned}
& T^{i j}=T_{l}^{i j}+T_{2 .}^{i j} \\
& J_{i j}=J_{i j}^{1}+J_{i j}^{2}+J_{i j}^{3}
\end{aligned}
$$

where,

$$
\begin{aligned}
& T_{1}^{i j}=g^{i j}\left\{\left(\widehat{P_{0}}\right)\left[\frac{(n P)}{(P k)}-\frac{\left(n P_{0}\right)}{\left(P_{0} k\right)}\right]-(n k)\left[\frac{1}{(P k)}+\frac{1}{\left(P_{0} k\right)}\right]\right\} \\
& T_{2}^{i j}=P_{0}^{i} k^{j} \frac{(P n)}{(P k)}+P^{i} k^{j} \frac{\left(P_{0} n\right)}{\left(P_{0} k\right)}-P^{i} P^{j} \frac{\left(n P_{o}\right)}{(P k)} \\
& +P_{0}^{i} P_{o}^{j} \frac{(P n)}{\left(P_{0} k\right)}-P^{i} P_{o}^{j}\left[\frac{\left(n P_{0}\right)}{(P k)}-\frac{(P n)}{\left(P_{0} k\right)}\right] \\
& J_{i j}^{l}=-\frac{\pi^{3}}{4\left(\vec{P}_{0} \times \vec{P}\right)^{2}}\left[\left\{\left(\frac{\left(\left(\vec{P}_{0} \times \vec{P}\right) \times \vec{P}_{o}\right)_{i}}{P_{0}} \frac{\left(P_{0} \tau_{j}+\tau P_{0 j}\right)}{\tau\left(P_{0} \tau+\vec{P}_{0} \cdot \vec{T}\right)}+i \hookrightarrow j\right)\right.\right.
\end{aligned}
$$

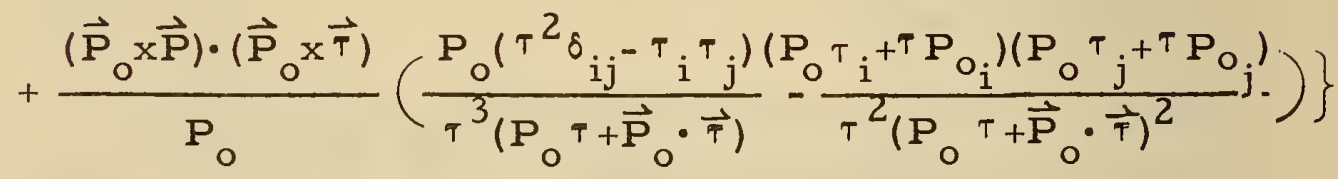

$$
\begin{aligned}
& \left.+\left\{\text { with } \vec{P}_{0} \zeta \vec{P}\right\}-\{\vec{\tau} \rightarrow \vec{q}\}-\left\{\vec{P}_{0} \zeta \vec{P} ; \vec{\tau} \rightarrow \vec{q}\right\}\right]
\end{aligned}
$$

$\overline{* c}$ f. the equations following equation 50 of reference (3). 


$$
\begin{aligned}
& J_{i j}^{2}=-\frac{\pi^{3}}{4\left(\vec{P}_{0} \times \vec{P}\right)^{2}}\left[\left\{\frac{\left(\vec{\tau} \cdot \vec{P}_{0} \times \vec{P}\right)^{2}\left(\tau^{2} \delta_{i j}-\tau_{i}{ }^{\tau} j\right.}{2 \tau^{3}(P \tau+\vec{P} \cdot \vec{\tau})\left(P_{0} \tau+\vec{P}_{0} \cdot \vec{\tau}\right)}\right\}\right. \\
& \left.+\left\{\vec{P}_{0} \hookrightarrow \vec{P}\right\}-\{\vec{T} \rightarrow \vec{q}\}-\left\{\vec{P}_{0} \hookrightarrow \vec{P}_{;} \vec{T} \rightarrow \vec{q}\right\}\right] \\
& J_{i j}^{3}=-\frac{\pi^{3}}{4\left(\vec{P}_{0} \times \vec{P}\right)^{2}}\left[\left\{\frac{-\left(P_{0} P \tau+P_{0} \vec{P} \cdot \vec{\tau}+P \vec{P}_{0} \cdot \vec{\tau}+\tau \vec{P}_{0} \cdot \vec{P}\right)}{2\left(P_{0} P+\vec{P}_{0} \cdot \vec{P}\right)^{2}(P \tau+\vec{P} \cdot \vec{\tau})^{2}\left(\tau P_{0}+\vec{\tau} \cdot \vec{P}_{0}\right)^{2}}\right.\right. \\
& x\left[\frac{1}{\tau}\left(P_{0} P+\vec{P}_{0} \cdot \vec{P}\right)\left(\vec{\tau} \times\left(\left(\vec{P}_{0} \times \vec{P}\right) \times \vec{\tau}\right)\right)_{i}+\left(\vec{\tau} \times\left(\left(\vec{P}_{0} \times \vec{P}\right) \times\left(P_{0} \vec{P}_{P} P_{P_{0}}\right)\right)\right)_{i}\right] \\
& \left.x[i \rightarrow j]\}+\left\{\vec{P}_{0} \leftarrow \vec{P}\right\}-\{\vec{T} \rightarrow \vec{q}\}-\left\{\vec{P}_{0} \leftarrow \vec{P} ; \vec{T} \rightarrow \vec{q}\right\}\right] \\
& \vec{R}=\frac{1}{2\left(P_{0} q+\vec{P}_{0} \cdot \vec{q}\right)}\left(\frac{\vec{P}_{0}}{P_{0}}+\frac{\vec{q}}{q}\right) \\
& \vec{S}=-\frac{1}{2(\vec{P} \times \vec{\tau})^{2}}\left\{\frac{1}{\tau} \vec{\tau} \times(\vec{P} \times \vec{\tau})-\frac{1}{q} \vec{q} \times(\vec{P} \times \vec{q})\right\} \\
& \vec{X}=\vec{P}\left[\frac{\left(P_{0} n\right)}{\left(P_{k} k\right.}+\frac{(k n)}{\left(P_{0} k\right)}+2\left(P_{0} n\right)+\frac{\left(P_{0} P\right)\left(P_{o} n\right)}{\left(P_{0} k\right)}+(P n)\right] \\
& +\vec{P}_{0}\left[\frac{\left(P_{0} n\right)}{(P k)}+\frac{(k n)}{\left(P_{0} k\right)}+\left(P_{0} n\right)+\frac{\left(P_{o} P\right)\left(P_{o} n\right)}{\left(P_{o} k\right)}-\frac{(k P)(k n)}{\left(P_{0} k\right)}\right] \\
& +\vec{k}\left[\frac{\left(P_{0} n\right)}{(P k)}-2 \frac{(P n)}{\left(P_{0} k\right)}+\left(P_{0} n\right)-\frac{\left(P_{o} P\right)(P n)}{\left(P_{0} k\right)}\right]
\end{aligned}
$$

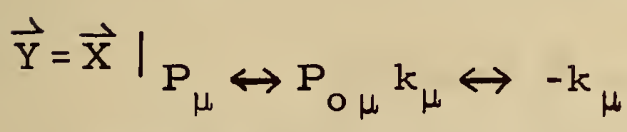

$$
\begin{aligned}
& \text { B. The Coplanar Case, } \overrightarrow{\mathrm{P}}_{\mathrm{o}} \cdot(\overrightarrow{\mathrm{P}} \times \overrightarrow{\mathrm{k}})=0
\end{aligned}
$$

The notation used here is the same as that for the coplanar case of the first order term (sec. 4-B).

$$
\text { It can be shown that } \begin{aligned}
T_{1}^{i j} J_{i j}^{1} & =0 \\
J_{i j}^{2} & =0 \\
\left(T_{1}^{i j}+T_{2}^{i j}\right) J_{i j}^{3} & =0
\end{aligned}
$$


The refore equation 5.2 becomes

$$
\chi=\left[\frac{2}{\pi^{3}} \mathrm{~T}_{2}^{\mathrm{ij}} \mathrm{J}_{\mathrm{ij}}^{1}+\frac{1}{(\mathrm{Pk})} \overrightarrow{\mathrm{R}} \cdot \overrightarrow{\mathrm{X}}-\frac{1}{\left(\mathrm{P}_{\mathrm{o}} \mathrm{k}\right)} \overrightarrow{\mathrm{S}} \cdot \overrightarrow{\mathrm{Y}}\right]
$$

For convenience the following are defined

$$
\begin{aligned}
& \omega_{1}=\frac{2}{\pi^{3}} T_{2}^{i j} J_{i j}^{1} \\
& \omega_{2}=\frac{1}{(P k)} \vec{R} \cdot \vec{X} \\
& \omega_{3}=-\frac{1}{\left(P_{0} k\right)} \vec{S} \cdot \vec{Y}
\end{aligned}
$$

then

$$
X=w_{1}+w_{2}+w_{3}
$$

i. Formulae for $w_{1}$

Two new terms $A_{i j}$ and $B_{i j}$ are defined from equation 5. 7:

$$
\begin{aligned}
& A_{i j}=\frac{1}{\left(\overrightarrow{P_{0}} \times \vec{P}\right)^{2}}\left[\left\{\frac{1}{P_{0}}\left\lfloor\left(\vec{P}_{0} \times \vec{P}\right) \times \vec{P}_{o}\right]_{i} \frac{\left(\tau P_{o j}+{ }^{\top}{ }_{j} P_{o}\right)}{\tau\left(P_{0} T+\vec{P}_{0} \cdot \vec{T}\right)}+i<j\right\}\right. \\
& \left.+\left\{\overrightarrow{\mathrm{P}}_{\mathrm{O}} \hookrightarrow \overrightarrow{\mathrm{P}}\right\}-\{\vec{\tau} \rightarrow \overrightarrow{\mathrm{q}}\}-\left\{\overrightarrow{\mathrm{P}}_{\mathrm{O}} \hookrightarrow \overrightarrow{\mathrm{P}} ; \vec{\tau} \rightarrow \overrightarrow{\mathrm{q}}\right\}\right] \\
& B_{i j}=\frac{1}{\left(\vec{P}_{0} \times \vec{P}\right)^{2}}\left[\left\{( \vec { P } _ { 0 } \times \vec { P } ) \cdot ( \vec { P } _ { 0 } \times \vec { \tau } ) \left[\frac{\left(\tau^{2} \delta_{i j}-\tau_{i} \tau_{j}\right)}{\tau^{3}\left(P_{0} \tau+\vec{P}_{0} \cdot \vec{\tau}\right)}\right.\right.\right. \\
& \left.\left.-\frac{\left(P_{o} \tau_{i}+\tau P_{o i}\right)\left(P_{o}{ }_{j}+\tau P_{o j}\right)}{P_{0} \tau^{2}\left(P_{o} \tau+\vec{P}_{\circ} \cdot \vec{\tau}\right)^{2}}\right]\right\} \\
& \left.+\left\{\overrightarrow{\mathrm{P}}_{\mathrm{O}} \hookrightarrow \overrightarrow{\mathrm{P}}\right\}-\{\vec{\tau} \rightarrow \overrightarrow{\mathrm{q}}\}-\left\{\overrightarrow{\mathrm{P}}_{\mathrm{O}} \hookrightarrow \overrightarrow{\mathrm{P}} ; \vec{\tau} \rightarrow \overrightarrow{\mathrm{q}}\right\}\right]
\end{aligned}
$$

Then $w_{1}$ can be expanded, noticing that $A_{i j}$ and $B_{i j}$ are symmetric where as $T_{2}^{i j}$ is not.

$$
\begin{aligned}
\omega_{1}= & -\frac{1}{2}\left[\mathrm{~T}_{2}^{11}\left(\mathrm{~A}_{11}+\mathrm{B}_{11}\right)+\left(\mathrm{T}_{2}^{12}+\mathrm{T}_{2}^{21}\right)\left(\mathrm{A}_{12}+\mathrm{B}_{12}\right)\right. \\
& \left.+\mathrm{T}_{2}^{22}\left(\mathrm{~A}_{22}+\mathrm{B}_{22}\right)\right]
\end{aligned}
$$

Equation 5.6 becomes after some algebra 


$$
\begin{aligned}
& T_{2}^{11}=\left[\frac{E P_{0} \cos \varphi_{0}}{E-P \cos \varphi}+\frac{E_{0} P \cos \varphi}{E E_{0}-P_{0} \cos \varphi_{0}}\right. \\
& \left.+\frac{\left(P_{0} \cos \varphi_{0}+P \cos \varphi\right)}{K}\left\{\frac{E P_{0} \cos \varphi_{0}}{E_{0} P_{0} \cos \varphi_{0}}-\frac{E D_{0} P \cos \varphi}{E-P \cos \varphi}\right\}\right] \\
& T_{2}^{12}+T_{2}^{21}=\left[\frac{E P_{0} \sin \varphi_{0}}{E-P \cos \varphi}+\frac{E_{0} P \sin \varphi}{E_{0}-P_{0} \cos \varphi_{0}}\right. \\
& +\frac{\left(P_{0} \cos \varphi_{0}+P \cos \varphi\right)}{K}\left\{\frac{E P_{0} \sin \varphi_{0}}{E_{0}-P_{0} \cos \varphi_{0}}-\frac{E{ }_{0} P \sin \varphi}{E-P \cos \varphi}\right\} \\
& \left.+\frac{\left(P_{0} \sin \varphi_{0}+P \sin \varphi\right)}{K}\left\{\frac{E P_{0} \cos \varphi_{0}}{E_{0}-P_{0} \cos \varphi_{0}}-\frac{E{ }_{0} P \cos \varphi}{E-P \cos \varphi}\right\}\right] \\
& T_{2}^{22}=\left[\frac{\left(P_{0} \sin \varphi_{0}+P \sin \varphi\right)}{K}\left\{\frac{E P_{0} \sin \varphi_{0}}{E_{0}-P_{0} \cos \varphi_{0}}-\frac{E{ }_{0} P \sin \varphi}{E-P \cos \varphi}\right\}\right]
\end{aligned}
$$

defined

In order to simplify the equations for the $A_{i j}$ 's the following are

$$
\begin{aligned}
& a=\left[P_{0} \tau\left(\tau+P_{0}-K \cos \varphi_{0}\right)\right]^{-1} \\
& b=\left[P \tau\left(\tau+P_{0} \cos \chi-K \cos \varphi\right)\right]^{-1} \\
& c=\left[P_{0} q\left(q+P_{0}-K \cos \varphi_{0}-P \cos \chi\right)\right]^{-1} \\
& d=\left[P q\left(q-P+P_{0} \cos \chi-K \cos \varphi\right)\right]^{-1}
\end{aligned}
$$

And

$$
\begin{aligned}
\tau^{2} & =\left(P_{0}^{2}+K^{2}-2 P_{0} K \cos \varphi_{0}\right) \\
q^{2} & =\left(\tau^{2}+P^{2}+2 P K \cos \varphi_{-} 2 P_{0} P \cos X\right)
\end{aligned}
$$

where equation 5.25 is the same as 4.4 . Then equation 5.17 becomes after some algebra 


$$
\begin{aligned}
& A_{11}=2\left[\frac{\left(\cos \varphi_{0} \cos \varphi-\sin \varphi_{0} \sin \varphi\right)}{P_{0} P}\left(\frac{1}{T}-\frac{1}{q}\right)\right. \\
& +\sin ^{2} \varphi_{0}\left\{c\left(1+\frac{K \sin \varphi_{0}}{P \sin \chi}\right)-a \frac{K \sin \varphi_{0}}{P \sin \chi}\right\} \\
& \left.-\sin ^{2} \varphi\left\{(d-b)\left(1+\frac{K \sin \varphi}{P_{0} \sin x}\right)\right\}\right] \\
& A_{12}=2\left[\frac{\left(\cos \varphi \sin \varphi_{0}+\cos \varphi_{0} \sin \varphi\right)}{P_{0} P}\left(\frac{1}{\tau}-\frac{1}{q}\right)\right. \\
& -\sin \varphi_{0} \cos \varphi_{0}\left\{c\left(1+\frac{K \sin \varphi_{0}}{P \sin \chi}\right)-a \frac{K \sin \varphi_{0}}{P \sin \chi}\right\} \\
& \left.+\sin \varphi \cos \varphi\left\{(d-b)\left(1+\frac{\mathrm{K} \sin \varphi}{\mathrm{P}_{0} \sin \chi}\right)\right\}\right]
\end{aligned}
$$

The equation for $A_{22}$ need not be given as it combines with $B_{22}$ in a rather simple manner

$$
\begin{aligned}
\left(A_{22}+B_{22}\right)=\left[-\left(A_{11}+B_{11}\right)\right. \\
\left.+\left\{c\left(1+\frac{K \sin \varphi_{0}}{P \sin \chi}\right)-a \frac{K \sin \varphi_{0}}{P \sin \chi}\right\}-\left\{(d-b)\left(1+\frac{K \sin \varphi}{P_{0} \sin \chi}\right)\right\}\right]
\end{aligned}
$$

In order to simplify the equations for the $B_{i j}$ 's the following are defined:

$$
\begin{aligned}
w= & \left(P_{0}+\tau\right) \cos \varphi_{0}-K \\
x= & P_{0} \cos \varphi_{0}+\tau \cos \varphi-K \\
y= & \left(P_{0}+q\right) \cos \varphi_{0}-P \cos \varphi-K \\
z= & P_{0} \cos \varphi_{0}-(P-q) \cos \varphi-K \\
\bar{w}= & \left(P_{0}+\tau\right) \sin \varphi_{0} \\
\bar{x}= & P_{0} \sin \varphi_{0}+\tau \sin \varphi \\
\bar{y}= & \left(P_{0}+q\right) \sin \varphi_{0}-P \sin \varphi \\
\bar{z}= & P_{0} \sin \varphi_{0}-(P-q) \sin \varphi \\
& P \operatorname{Pin}_{0}^{2} \varphi_{0} \\
L= & \frac{\tau^{2}}{}
\end{aligned}
$$




$$
\begin{aligned}
& M=\frac{\left(P_{0} \sin \varphi_{0}-P \sin \varphi\right)^{2}}{q^{2}} \\
& \bar{L}=\frac{P_{0} \sin \varphi_{0}\left(P_{0} \cos \varphi_{0}-K\right)}{\tau^{2}} \\
& \bar{M}=\frac{\left(P_{0} \sin \varphi_{0}-P \sin \varphi\right)\left(P_{0} \cos \varphi_{0}-P \cos \varphi-K\right)}{q^{2}}
\end{aligned}
$$

By use of equation 5.28 , only the $B_{11}$ and $B_{12}$ terms need be given.

$$
\begin{aligned}
& B_{11}=\left[a \frac{K \sin \varphi_{0}}{P \sin \chi}\left\{P_{0} a w^{2}-L\right\}\right. \\
& -b\left(1+\frac{K \sin \varphi}{P_{0} \sin x}\right)\left\{P b x^{2}-L\right\} \\
& -c\left(1+\frac{K \sin \varphi_{0}}{P \sin \chi}\right)\left\{P_{0} c y^{2}-M\right\} \\
& \left.+d\left(1+\frac{K \sin _{\varphi}}{P_{0} \sin \chi}\right)\left\{P d z^{2}-M\right\}\right] \\
& B_{12}=\left[a \frac{K \sin \varphi_{0}}{P \sin \chi}\left\{P_{0} a w \bar{w}+\bar{L}\right\}\right. \\
& -b\left(1+\frac{K \sin \varphi}{P_{0} \sin \chi}\right)\{P b x \bar{x}+\bar{L}\} \\
& -c\left(1+\frac{K \sin \varphi_{0}}{P \sin \chi}\right)\left\{P_{0} c y \bar{y}+\bar{M}\right\} \\
& \left.+d\left(1+\frac{K \sin \varphi}{P_{0} \sin \chi}\right)\{P d z \bar{z}+\bar{M}\}\right]
\end{aligned}
$$

ii. Formulae for $\omega_{2}$ 


$$
\begin{aligned}
\omega_{2} & =-\frac{1}{2} \frac{C}{(E-P \cos \varphi)}\left\{\frac{P}{K}\left[\left(P_{0}+q\right) \cos \chi-P-K \cos \varphi\right] C_{1}\right. \\
& +\frac{P_{0}}{K}\left[\left(P_{0}+q\right)-P \cos \chi-K \cos \varphi_{0}\right] C_{2} \\
& \left.+\left[\left(P_{0}+q\right) \cos \varphi_{0}-P \cos \varphi-K\right] C_{3}\right\}
\end{aligned}
$$

where

$$
\begin{aligned}
& C_{1}=\{-E_{0}\left[3+\frac{\left(E E_{0} E-P_{0} P \cos \chi\right)}{K\left(E_{0}-P_{0} \cos \varphi_{0}\right)}-\frac{1}{K(E-P \cos \varphi)}\right] \\
&\left.+K+\frac{1}{\left(E_{0}-P_{0} \cos \varphi_{0}\right)}\right\} \\
& C_{2}=C_{1}+E_{0}+E+K \frac{(E-P \cos \varphi)}{\left(E-P_{0} \cos \varphi_{0}\right)} \\
& C_{3}=\left\{E_{0}\left[\frac{1}{K(E-P \cos \varphi)}-1\right]-E\left[\frac{2-\left(E_{0} E-P_{0} P \cos \chi\right)}{K\left(E_{0}-P_{0} \cos \varphi_{0}\right)}\right]\right\}
\end{aligned}
$$

The $c$ in equation 5.32 is the c defined in equations 5.23. iii. Formulae for $w_{3}$

$$
\begin{aligned}
& \omega_{3}=-\frac{1}{2} \frac{1}{K\left(E_{0}-P_{0} \cos \varphi_{0}\right)}\left\{\frac{\left[\left(\frac{1}{\tau}-\frac{1}{q}\right) \frac{P_{0} K \sin \varphi_{0}}{P}-\frac{P_{0}}{q} \sin x\right] C^{1}}{\left(P_{0} \sin \chi+K \sin \varphi\right)}\right. \\
& \left.+\left(\frac{1}{\tau}-\frac{1}{q}\right) C^{2}-\frac{\left[\left(\frac{1}{\tau}-\frac{1}{q}\right) \frac{P_{0} K \sin \varphi_{0}}{P}+\frac{K}{q} \sin \varphi\right] C^{3}}{\left(P_{0} \sin \chi+K \sin \varphi\right)}\right\}
\end{aligned}
$$

where

$$
\begin{aligned}
C^{1}= & -E\left\{3+\frac{1}{K\left(E E_{0}-P_{0} \cos \varphi_{0}\right)}-\frac{\left(E_{0} E-P_{0} P \cos \chi\right)}{K(E-P \cos \varphi)}\right\}-K \\
& \left.+\frac{1}{(E-P \cos \varphi)}\right]
\end{aligned}
$$




$$
\begin{aligned}
& C^{2}=C^{1}+E_{0}+E-\frac{K\left(E_{0}-P_{0} \cos \varphi_{0}\right)}{(E-P \cos \varphi)} \\
& C^{3}=\left[-E\left\{1+\frac{1}{K\left(E_{0}-P_{0} \cos \varphi_{0}\right)}\right\}+E_{0}\left\{\frac{2-\left(E_{0} E-P_{0} P \cos \chi\right)}{K(E-P \cos \varphi)}\right\}\right]
\end{aligned}
$$

6. Discussion of the Second Order Coplanar Equation

It should be noted that the re occur several points in the equations for the second order term that are of the form $\frac{0}{0}$ (for example, when $\sin x=0$ in the equation for $\omega_{1}(5.19)$ and when $P_{0} \sin x+k \sin \varphi=0$ in the equation for $\omega_{3}(5.36)$ ). However, the value of $\psi_{2}$ is finite at each of these points.

The calculation of the limiting values is rather involved and will not be given here. 
We consider the following case where there is a considerable difference between the first order theory and experimental values. Thus the second order term would be expected to be significant.

$$
\begin{aligned}
& \mathrm{T}_{\mathrm{o}}=500 \mathrm{kev} \\
& \mathrm{k}=450 \mathrm{kev} \\
& \varphi_{0}=20^{\circ}
\end{aligned}
$$

TABLE I

$\varphi \quad \psi_{1} \frac{\mathrm{mb}}{\text { ster/ster }}$

$$
\psi_{2} \frac{\mathrm{mb}}{\text { ster/ster }} \frac{\mathrm{kd} \sigma \mathrm{Al}}{\mathrm{z}^{2} \mathrm{dkd} \Omega \mathrm{p}^{\mathrm{d} \Omega_{\mathrm{k}}}} \frac{\mathrm{kd} \sigma \mathrm{Au}}{\mathrm{z}^{2} \mathrm{dkd} \Omega \mathrm{p} \Omega_{\mathrm{k}}}
$$

$\begin{array}{ll}80^{\circ} & .050 \\ 60^{\circ} & .125 \\ 40^{\circ} & .280 \\ 25^{\circ} & .357 \\ 15^{\circ} & .355 \\ 0^{\circ} & .295 \\ -20^{\circ} & .195 \\ -60^{\circ} & .075\end{array}$

.0016

.0043

.0090

.0115

.0114

.0095

.0062

.0025
.071

.178

.397

.506

.503

.419

.276

.108
.180

.449

.991

1.26

1. 26

1.05

.687

.273

Values for $\psi_{1}$ and $\psi_{2}$ are plotted in Figure 1. 


\section{Summary}

The contribution to the differential cross section by the second order term for the case calculated in section 7 leads to an increase of about $50 \%$ for $\mathrm{Al}$ and about $200 \%$ for $\mathrm{Au}$ as would be expected as discussed in section 1. However, these values cannot be compared with existing experimental data since these data are for the integrated (over finial electron direction) cross sections.

Since the addition from the second order term (at least for the above case) is so large, it appears that the third or even higher order terms should be calculated or estimated as to magnitude and algebraic sign. The case might be that the first order term of the series gives a closer approximation to the limiting value of the series than the sum of the first several terms. Or, conversely, the third and higher order terms might make a negligible contribution.

Therefore, since at the present time a third order term has not been calculated and no experimental data for the cross section differential with respect to both final electron and photon directions are available, it cannot be said exactly what the limitations on this second order contribution are.

Although, as pointed out above, the values obtained in section 7 cannot be compared to experimental data, a rough approximation to the integrated cross-section for this case only can be made. It is noticed that even in the non-coplanar case $\psi_{2}$ is the sum of two parts (see equation 5.1), one involving $X$ (dependent on the final electron direction), the other, a multiplicative factor of $\psi_{1}$, independent of angles $i_{\bullet}$ e. $\pi \propto\left(\frac{E}{P}-\frac{E_{0}}{P_{0}}\right)$ which is always positive. In the case tabulated the former is approximately $1 / 10$ of the latter for all final electron angles. Therefore if it is assumed that the relationship between the two does not change much as the plane containing $\vec{P}$ is rotated about $\vec{P}_{0}$ then the integral of $\psi_{2}$ over final electron direction 


$$
\begin{aligned}
\int \psi_{2} d \Omega_{p} & =\left\{\pi \alpha\left(\frac{E}{p}-\frac{E_{o}}{P_{o}}\right) \int \psi_{1} d \Omega_{p}+\right. \\
& \left.+\frac{\alpha^{2}}{2 \pi} r_{0}^{2} \frac{P_{k}^{2}}{P_{o}} \int \frac{\chi}{q^{2}} d \Omega_{p}\right\}
\end{aligned}
$$

is the sum of two positive quantities that are approximately in the ratio of 10 to 1 .

$$
\text { Therefore } \int \psi_{2} d_{\Omega}>\pi \alpha\left(\frac{E}{P}-\frac{E_{o}}{P_{o}}\right) \int \psi_{1} d \Omega_{p}
$$

Then from equation 3.1

$$
\int \mathrm{d} \sigma \mathrm{d} \Omega_{\mathrm{p}}=\mathrm{z}^{2} \frac{\mathrm{dk}}{\mathrm{k}} \mathrm{d} \Omega_{\mathrm{k}} \int\left(\psi_{1}+\mathrm{Z}_{\psi_{2}}\right) \mathrm{d} \Omega_{\mathrm{p}}
$$

Substitution of 8.2 into 8.3 gives

$$
\int d \sigma d \Omega_{p}>\left\{z^{2} \frac{d k}{k} d \Omega_{k} \int \psi_{1} d \Omega_{p}\right\}\left\{1+\pi \alpha z\left(\frac{E}{P}-\frac{E_{o}}{P_{o}}\right\}\right.
$$

Let

$$
\begin{aligned}
& \mathrm{d} \sigma(1 \mathrm{st})=\mathrm{z}^{2} \frac{\mathrm{dk}}{\mathrm{k}} \mathrm{d} \Omega_{\mathrm{k}} \int_{\psi_{1}} \mathrm{~d} \Omega_{\mathrm{p}} \\
& \mathrm{d} \sigma(1 \mathrm{st}+2 \mathrm{~d})=\mathrm{z}^{2} \frac{\mathrm{dk}}{\mathrm{k}} \mathrm{d} \Omega_{\mathrm{k}} \int_{\left(\psi_{1}+\mathrm{Z}_{\psi_{2}}\right) \mathrm{d} \Omega_{\mathrm{p}}} \\
& \mathrm{d} \sigma_{\text {exp }}=\text { the experimental value for the integrated cross section. }
\end{aligned}
$$

Then 8.4 becomes upon substitution of numerical values

$$
\mathrm{d} \sigma(1 \mathrm{st}+2 \mathrm{~d})>1.029 \mathrm{Zd} \sigma(1 \mathrm{st})
$$

In reference (1) experimental values are given for $\mathrm{T}_{\mathrm{o}}=500 \mathrm{kev}$, $k=450 \mathrm{kev} ; \theta_{0}=0^{\circ}, 30^{\circ}, 60^{\circ}$, and $90^{\circ}$ for $\mathrm{Z}=13$ and 79 , with and estimated error within $20 \%$. Interpolation for $\Theta_{0}=20^{\circ}$ gives approximately: 
TABLE II

$$
\mathrm{Z}=13 \quad \mathrm{Z}=79
$$

\begin{tabular}{|l|l|l|}
\hline$\frac{\mathrm{k} d \sigma}{\mathrm{z}^{2} \mathrm{dkd} \Omega_{\mathrm{k}}} \exp$ & $2.0 \pm 0.4 \frac{\mathrm{mb}}{\mathrm{ster}}$ & $3.2 \pm 0.6 \frac{\mathrm{mb}}{\mathrm{ster}}$ \\
\hline$\frac{\mathrm{d} \sigma}{\mathrm{d} \sigma(1 \mathrm{st})} \exp$ & $4.0 \pm 0.8$ & $6.4 \pm 1.3$ \\
\hline$\frac{\mathrm{d} \sigma(1 \mathrm{st}+2 \mathrm{~d})}{\mathrm{d} \sigma(1 \mathrm{st})}$ & $>1.38$ & $>3.29$ \\
\hline$\frac{\mathrm{d} \sigma}{\mathrm{d} \sigma(1 \mathrm{st}+2 \mathrm{~d})}$ & $<2.9 \pm 0.6$ & $<1.9 \pm 0.4$ \\
\hline
\end{tabular}

therefore, even with this crude approximation of the integrated cross section, the second order term brings the Born approximation into better agreement with existing experimental data, (at least for the particular case as tabulated in section 7). 


\section{References}

(1). J. W. Motz, Phys. Rev. 100, pg. 1560 (1955).

(2). H. W. Koch and J. W. Motz, Rev. M. Physics, 31, pg. 920 (1959).

(3). C. Kacser, Proc. Roy. Soc., A253, pg. 103 (1959). 


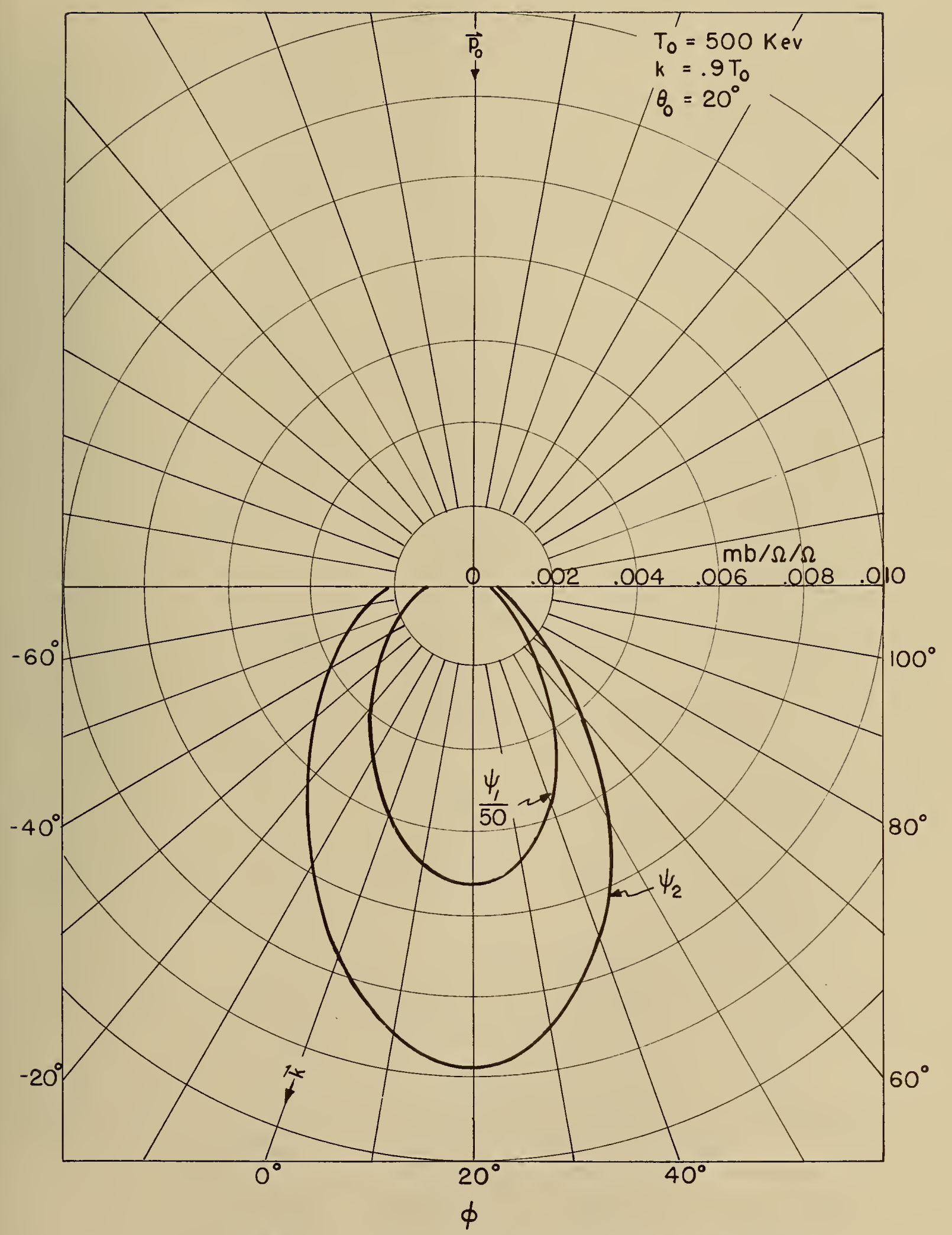

Fig. 1. Variation of $\Psi_{1}$ and $\Psi_{2}$ with angle, $\varphi$, from the data of TABLE I. 



\section{U. S. DEPARTMENT OF COMMERCE \\ Luther H. Hodges, Secretary}

NATIONAL BUREAU OF STANDARDS

A. V. Astin, Director

\section{THE NATIONAL BUREAU OF STANDARDS}

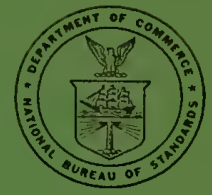

The scope of activitics of the National Bureau of Standards at its najor lahoratories in Waslington, D.C., and Boulder, Colorado, is suggested in the following listing of the divisions and sections engaged in technical work. In general, each section carries out specializcd research, development, and engineering in the field indicated by its title. A brief description of the activitics, and of the resultant publications, appears on the inside of the front cover.

\section{WASHINGTON, D.C.}

Flectricity. Resistance and Rcactance. Electrochenistry. Electrical Instruments. Magnetic Measırements. Diclectrics.

Metrology. Photomctry and Colorimetry. Refiactometry. Photographic Researclı. Length. Engineering Metiology. Mass and Seale. Volunetry and Densimetry.

Heat. Temperature Physies. Heat Measurements. Cryogenic Physics. Equation of State. Statistical Physics. Radiation Physics. X-ray. Radioactivity. Radiation Theory. High Energy Radiation. Radiological Equipment. Nucleonic Instrumentation. Nentron Physics.

Analytical and Inorganic Chemistry. Pure Substances. Spectrochemistry. Solution Chemistry. Analytical Chenistry. Inorganic Chemistry.

Mechanics. Sound. Pressurc and Vacuum. Fluid Mechanics. Engineering Mechanics. Rhcology. Combustion Controls.

Organic and Fibrous Materials. Rubber. Textiles. Paper. Leather. Testing and Specifications. Polyner Structure. Plastics. Dental Rescarch.

Metallırgy. Thermal Metallurgy. Chenical Metallurgy. Mechanical Metallurgy. Corrosion. Metal Physics Mineral Products. Engineering Ceramics. Glass. Refractorics. Enameled Mctals. Crystal Growtl.

Physical Properties. Constitution and Microstructure.

Building Research. Structural Engineering. Fire Rescarclı. Mcchanical Systeuns. Organic Building Materials. Codes and Safcty Standards. Meat Transfer. Inorganic Building Materials.

Applied Mathematics. Numerical Analysis. Computation. Statistical Engineering. Mathematical Physics.

Data Processing Systems. Components and Teclnniques. Digital Circuitry. Digital Systems. Analog Systenıs. Applications Engineering.

Atomic Physics. Spectroscopy. Radiometry. Solid State Physics. Electron Physics. Atomic Physics.

Instrumentation. Enginecring Electronics. Electron Devices. Electronic Instrumentation. Mechanical Instru. ments. Basic Instrumentation.

Physical Chemistry. Thernochenistry. Surface Chemistry. Organic Chemistry. Molecular Spectroscopy. Molecular Kinetics. Mass Spectrometry. Molecular Structure and Radiation Chemistry.

- Office of Weiglits and Measures.

\section{BOULDER, COLO.}

Cryogenic Engineering. Cryogenic Equipment. Cryogenic Processcs. Propertics of Materials. Gas Liquefaction. Ionosphere Research and Propagation. Low Frcquency and Very Low Frequency Research. Ionosphere Rescarcl. Prediction Services. Sun-Earth Relationships. Field Engineering. Radio Warning Services.

Radio Propagation Engineering. Data Reduction'Instrumentation. Radio Noise. Tioposplicric Measurenents. Tropospheric Analysis. Propagation-Terrain Effccts. Radio-Meteorology. Lower Atnospluete Physics.

Radio Standards. Higl Frequency Electrical Standards. Radio Broadcast Service. Radio and Microwave Materials. Atomic Frequency and Time Interval Standards. Electronic Calibration Center. Millineter-Wave Research. Micıowave Circuit Standards.

Radio Systenıs. Iligh Freqnency aud Very High Frcqucncy Research. Modulation Reseatch. Antenna Research. Navigation Systems. Space Telecommunications.

Cpper Atmosphere and Space Physies. Upper Atmosphete and Plasina Physics. Ionosphere and Exosphere Scatter. Airglow and Aurora. Ionospheric Radio Astronomy. 
